\title{
Optical Properties of Some Compound Semiconductors in the 36-150-eV Region
}

\author{
Manuel Cardona* and Ruprecht Haensel \\ Deutsches Elektronen-Synchrotron, Hamburg, Germany \\ and \\ II. Institut für Experimental-Physik der Universität \\ Hamburg, Hamburg, Germany
}

(Received 3 November 1969)

\begin{abstract}
The absorption spectra of evaporated thin films of $\mathrm{ZnS}, \mathrm{CdS}, \mathrm{ZnSe}, \mathrm{CdSe}, \mathrm{ZnTe}, \mathrm{CdTe}, \mathrm{PbS}$, and $\mathrm{PbTe}$ in the $36-150-\mathrm{eV}$ region are reported. The measurements were performed using the DESY electron synchrotron as a radiation source. A sharp doublet associated with transitions from the $3 p$ core levels of $\mathrm{Zn}$ to the conduction bands $(3 p \rightarrow c)$ is observed for $\mathrm{ZnS}, \mathrm{ZnSe}$, and less clearly for $\mathrm{ZnTe}$. The splitting of this doublet seems due to spin-orbit interaction. Other less pronounced structure (Se: $3 d \rightarrow c$ in $\mathrm{ZnSe}$, $\mathrm{Cd}: 4 p \rightarrow c$ in $\mathrm{CdTe}$ ) is also observed, but most spectra are dominated by broad transitions similar to the atomic $d \rightarrow f$ transitions observed in this region for $\mathrm{Kr}$ and $\mathrm{Xe}$. The number of effective electrons whose oscillator strength has been exhausted at a given energy is obtained by integrating the absorption coefficient. In the compounds containing the heavy elements $\mathrm{Te}$ and $\mathrm{Pb}$, all outermost $d$ electrons of the core have exhausted their oscillator strength at $150 \mathrm{eV}$.
\end{abstract}

\section{INTRODUCTION}

$T$ HE intrinsic optical properties of the IIb-VIb family of semiconductors and insulators with zinc-blende and wurtzite structure have been the object of considerable study. ${ }^{1-3}$ The measurements, usually performed on single crystals with the normal-incidence reflection technique, have been confined to the region of photon energies below $30 \mathrm{eV}^{4-6}$ Transmission measurements with vacuum-deposited polycrystalline samples, ${ }^{7}$ limited because of substrate absorption to the $1-6-\mathrm{eV}$ energy region, have shown substantial agreement with reflectivity measurements on bulk single crystals.

In the $1-10-\mathrm{eV}$ region, the optical properties of the IIb-VIb materials are dominated by direct interband transitions between the valence and conduction bands. The absorption coefficient has an absolute maximum in the near ultraviolet (around $6 \mathrm{eV}$ ) where most of the oscillator strength is concentrated. The band-structure assignment of this maximum, usually labeled $E_{2}$, has been extensively discussed in the literature. ${ }^{2}$ Additional structure is observed at both sides of this maximum; we shall mention in particular the $E_{1}, E_{1}+\Delta_{1}$ peaks, split by spin-orbit interaction and located at photon energies below $E_{2}$. The line shape of these peaks is known to be strongly affected by exciton in teraction. ${ }^{8}$ Exciton effects are also responsible for the rich structure observed in the neighborhood of the fundamental edge. ${ }^{1}$

* John S. Guggenheim Memorial Foundation Fellow, on leave from Brown University, Providence, R. I.

1 T. C. Collins, C. W. Litton, and D. C. Reynolds, Phys. Status Solidi 12, 3 (1965).

${ }^{2}$ F. H. Pollak, in II-VI Semiconducting Compounds, edited by D. G. Thomas (W. A. Benjamin Inc., New York, 1967), p. 552; M. L. Cohen, ibid., p. 462.

${ }^{3}$ F. Herman, R. L. Kortum, C. D. Kuglin, and J. L. Shay, in II-VI Semiconducting Compounds, edited by D. G. Thomas (W. A. Benjamin, Inc., New York, 1967), p. 503.

${ }^{4}$ M. Cardona and D. L. Greenaway, Phys. Rev. 131, 98 (1963).

5 M. Aven, D. T. F. Marple, and B. Segall, J. Appl. Phys. 32S, 2261 (1961).

${ }^{6}$ W. J. Scouler and G. B. Wright, Phys. Rev. 133, A736 (1964).

${ }^{7}$ M. Cardona and G. Harbeke, J. Appl. Phys. 34, 813 (1963).

${ }^{8}$ E. O. Kane, Phys. Rev. 180, 852 (1969).
In the 10-30-eV region, transitions from the outermost $d$ electrons of the cation core into the conduction band are observed. ${ }^{4}$ These transitions are centered around $15 \mathrm{eV}$ and have an approximate width of $4 \mathrm{eV}$. Fine structure in these transitions has been observed ${ }^{9}$ for Cd but most of the reported data show a single peak. However, structure at the $d$ peaks could have been missed in these experiments because of the inadequacy of the discrete line sources conventionally used. Such structure would be related to structure in the density of conduction states and to the spin-orbit splitting of the $d$-core levels: The bands formed by these levels should have negligible width other than lifetime broadening. ${ }^{10}$ The relationship between the spectrum of the $3 d \rightarrow c$ transitions of $\mathrm{Ge},{ }^{11}$ a material closely related to the II-VI compounds, and the density of conduction states has been pointed our recently. ${ }^{12}$

The intrinsic optical properties of the lead chalcogenides (IVb-VIb compounds with rocksalt structure) have also been extensively studied. Reflectivity measurements have been performed up to $25 \mathrm{eV},{ }^{13}$ while transmission measurements on vacuum-deposited films have been confined to energies below $6 \mathrm{eV} .^{13,14}$ Transmission measurements are particularly attractive for these materials because of the possibility of preparing high-quality single-crystal films by epitaxial deposition on cleaved alkali-halide substrates. ${ }^{14}$

The results of transmission measurements on lead chalcogenides are in essential agreement with those of reflectivity measurements. They show structure presumably due to valence-to-conduction-band transitions

${ }^{9}$ W. C. Walker and J. Osantowski, J. Phys. Chem. Solids 25, 778 (1964).

${ }^{10} \mathrm{P}$. Eckelt, O. Madelung, and J. Treusch, Phys. Rev. Letters 18, 656 (1967).

${ }_{11}$ B. Feuerbacher, M. Skibowski, R. P. Godwin, and T. Sasaki, J. Opt. Soc. Am. 58, 1434 (1968).

${ }_{12} \mathrm{M}$. Cardona, in Proceedings of the Conference on Electronic Density of States, Gaithersburg, Maryland, 1969 (to be published).

${ }_{13}^{13}$ M. Cardona and D. L. Greenaway, Phys. Rev. 133, A1685 (1964).

${ }^{14}$ P. R. Wessel, Phys. Rev. 153, 836 (1967). 
below $20 \mathrm{eV}$ and the beginning of structure due to transitions from the outermost $d$ electrons of $\mathrm{Pb}$ above $20 \mathrm{eV}$. The absorption coefficient shows a broad absolute maximum around $3 \mathrm{eV}\left(E_{2}\right)$ with some weak structure before and after this maximum. This structure is broader and not as well defined as that observed for the zinc blende family; hence, the band-structure assignment of the observed peaks has remained somewhat uncertain. Optical-constants calculations from band structure, such as those performed recently by Buss and Parada ${ }^{15}$ for $\mathrm{PbTe}$, should contribute to the identification of the observed structure.

In this paper, we report measurements of the absorption coefficient of $\mathrm{ZnS}, \mathrm{CdS}, \mathrm{ZnSe}, \mathrm{CdSe}, \mathrm{ZnTe}$, CdTe, $\mathrm{PbS}$, and $\mathrm{PbTe}$ at room temperature in the $36-150-\mathrm{eV}$ region. The measurements were performed on films vacuum-deposited on thin carbon substrates. The choice of materials was determined, in part, by the fact that they yield reasonably crystalline films when deposited on substrates at room temperature. From our experience with these films in the visible and near uv, we can state that the width of the structure observed in the 36$150-\mathrm{eV}$ region $(>1 \mathrm{eV})$ is not determined by crystalline imperfections in our films. The transmission technique was chosen over that of normal-incidence reflection because of the small reflectivity of our materials $(<0.01)$ in the 36-150-eV region, which increases the error due to scattering light of long wavelengths. Most of the structure observed is rather broad and is believed related to the $d \rightarrow f$ structure observed in atomic spectra, ${ }^{16}$ in the solid rare gases, ${ }^{17}$ and in the alkali iodides. ${ }^{18}$ Sharp doublet structure has been observed for the $\mathrm{Zn}$ compounds, especially $\mathrm{ZnS}$ and $\mathrm{ZnSe}$. This structure is related to transitions from the outermost core levels of $\mathrm{Zn}$ to the conduction band and exhibits a splitting equal to that expected for these $d$ levels (spin-orbit splitting $\approx 3 \mathrm{eV}^{19}$ ). The absorption spectrum of $\mathrm{ZnSe}$ exhibits also sharp structure related to transitions originating at the outermost $3 d$ levels of Se.

\section{EXPERIMENTAL PROCEDURE}

The absorption experiments were performed using as a radiation source the DESY electron synchrotron..$^{20,21}$ For the standard synchrotron operating conditions (final energy between 3 and $7 \mathrm{GeV}$ ), the photon flux on the entrance slit is $10^{12}$ photons/ $\left(\mathrm{sec} \mathrm{eV} \mathrm{cm}^{2}\right)$ at $100-\mathrm{eV}$ photon energy. The samples were placed in front of the entrance slit of a Rowland grazing-incidence mono-

${ }^{15}$ D. Buss and N. J. Parada, Phys. Rev. (to be published).

${ }_{16}$ U. Fano and J. W. Cooper, Rev. Mod. Phys. 40, 441 (1968).

${ }_{17}$ R. Haensel, G. Keitel, P. Schreiber, and C. Kunz, Phys. Rev. $188,1375(1969)$.

${ }^{18} \mathrm{H}$. Fujita, C. Gähwiller, and F. C. Brown, Phys. Rev. Letters 22, $1369(1969)$.

${ }_{19}$ F. Herman and S. Skillman, Atomic Energy Levels (PrenticeHall, Inc., Englewood Cliffs, N. J., 1963).

${ }_{20}$ R. Haensel and C. Kunz, Z. Angew. Phys. 23, 276 (1967).

${ }^{21}$ R. P. Godwin, in Springer Tracts in Modern Physics, edited by G. Höhler (Springer-Verlag, Berlin, 1969), Vol. 51, p. 1. chromator. A gold-coated grating with 2400 lines/mm and a grazing angle of incidence of $4^{\circ} 16^{\prime}$ was used in first order with an angle of incidence of $12^{\circ} 30^{\prime}$; it provided an average resolution of $0.1 \AA$ with $20-\mu \mathrm{m}$ slits. The detector was a Bendix M 306 open magnetic electron multiplier.

The photomultiplier response in the absence of a sample had a maximum at a photon energy of about $100 \mathrm{eV}$ due, in part, to the blazing of the grating and to the spectral distribution of the synchrotron radiation. As a result of the rapid decrease in the photon flux below $100 \mathrm{eV}$, the influence of higher-order radiation at a first-order setting of $50 \mathrm{eV}$ is not negligible. In order to avoid this problem, we used as filters unsupported Al films about $1500 \AA$ A thick. The sharp absorption edge of this metal at $72.7 \mathrm{eV}$ ensures virtually pure first-order radiation between 36 and $72 \mathrm{eV}$. Measurements at $72 \mathrm{eV}$ with and without filter indicate a tolerable amount of higher-order radiation $(\leqslant 5 \%)$ at this and higher energies. The upper photon energy limit of our measurements was determined by the small radiation intensity available with the small grazing angle required and the beginning of the scattered light associated with the zero-order spectrum.

The samples were prepared by vacuum deposition at pressures about $10^{-6} \mathrm{~T}$ in situ and $e x$ situ. The substrates were $20-\mu \mathrm{g} / \mathrm{cm}^{2}$ carbon films supported by a $70-\mu m$ copper grid; they were kept at room temperature during evaporation. The film thickness was determined while evaporating with a quartz film-thickness monitor. After evaporation, the thickness of a film deposited on a glass substrate, placed next to the carbon substrate during evaporation, was measured by the Tolansky method: Results consistent to better than $20 \%$ with those of the quartz monitor were found. The results of the Tolansky measurements were used to evaluate the absorption coefficients, since they were thought to be more reliable than those of the quartz monitor. From the consistency of the results obtained for several samples, we believe, however, that the absorption coefficients reported for $\mathrm{CdSe}, \mathrm{ZnSe}, \mathrm{CdTe}$, and $\mathrm{ZnTe}$ are affected by a scaling error of about $20 \%$ due to uncertainties in the thickness determination. This error may be as high as $30 \%$ for $\mathrm{ZnS}$ and $\mathrm{CdS}$.

As already mentioned, measurements were performed both with samples deposited on the substrates in situ and with samples prepared in an external evaporator; no differences were found between samples of the same material prepared by either procedure. In situ preparation has the advantage of permitting the measurements of the transmissivity of the substrate immediately before deposition. This feature becomes important when the sample transmissivity is large.

\section{RESULTS}

Figure 1 shows the absorption spectra of $\mathrm{CdS}$ and $\mathrm{ZnS}$ at room temperature. This figure represents the 
Fig. 1. Absorption spectra of $\mathrm{ZnS}$ and $\mathrm{CdS}$ at room temperature in the soft-x-ray region. In this and subsequent figures, the vertical scale on on the left represents megabarns per diatomic molecule and is common to both compounds. The vertical scales on the right represent absorption coefficients and are different for each compound.

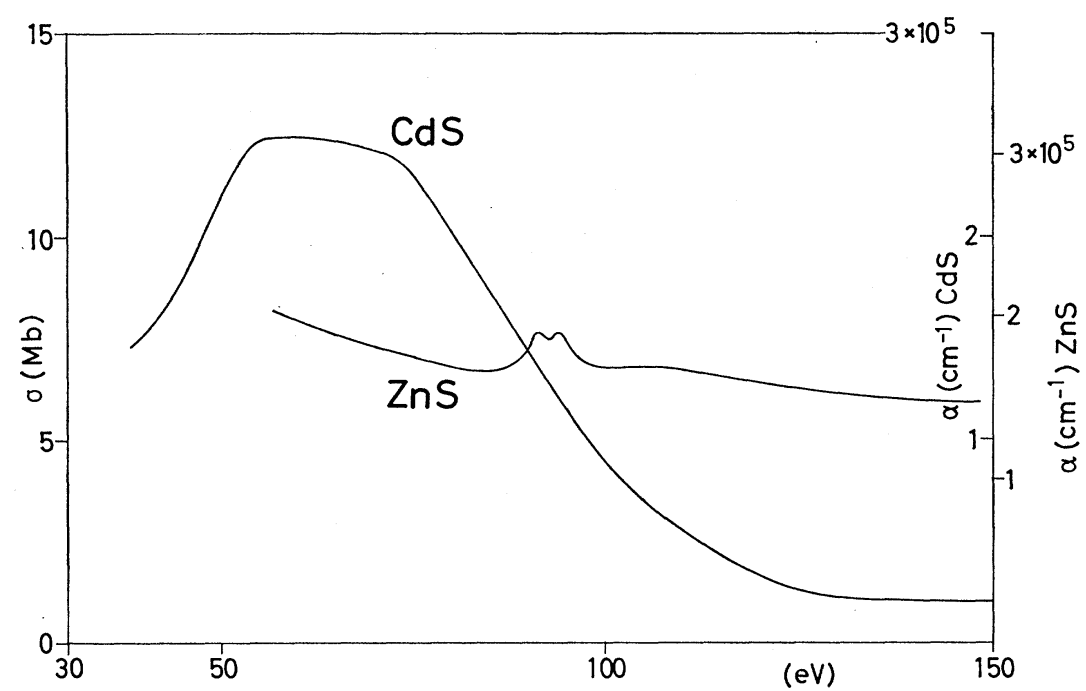

The absorption spectra of $\mathrm{ZnSe}$ and CdSe are shown in Fig. 2. Like in Fig. 1, the absorption of the cadmium compound below $90 \mathrm{eV}$ is stronger than that of $\mathrm{ZnSe}$. This fact will be discussed quantitatively in Sec. IV. The cadmium compound shows also only broad structure with a maximum at an energy slightly higher than that of CdS. The spectrum of $\mathrm{ZnSe}$ shows also the doublet which we have associated with the $M_{\text {II,III }}$ edge of $\mathrm{Zn}$. A slightly broader peak is observed at $59 \mathrm{eV}$ : Comparison with calculations of atomic levels ${ }^{19}$ and soft-x-ray emission data ${ }^{22}$ suggests that this peak is related to the $M_{\mathrm{IV}, \mathrm{V}}$ edge of Se (see Table I). The spin-orbit splitting expected for this edge $(\sim 1 \mathrm{eV})$ is too small to be resolved with the linewidth observed for this peak $(\sim 6 \mathrm{eV}$, not resolution limited).

We show in Fig. 3 a blown-up version of the $M_{\text {II,III }}$ peaks of $\mathrm{ZnS}$ and $\mathrm{ZnSe}$. For the purpose of the discussion in Sec. IV, we include in this figure the density of conduction states calculated for $\mathrm{ZnS}$ by Herman et al. ${ }^{3}$ As shown in this figure and in Table $I$, this doublet occurs in $\mathrm{ZnSe}$ at an energy $0.3-0.4 \mathrm{eV}$ lower than in $\mathrm{ZnS}$. In this regard, it is interesting to point out that

TABle I. Position (in eV) of "sharp" peaks identified in the soft-x-ray spectra of $\mathrm{ZnS}, \mathrm{ZnSe}, \mathrm{ZnTe}$, and CdTe. Also, corresponding emission thresholds reported by Bearden and Burr (Ref. 22) and binding energies of the assigned core levels calculated by Herman and Skillman (Ref. 19). For convenience, we use in this paper both the atomic and the x-ray level assignments for thresholds and edges, i.e., $K$ corresponds to $1 s, L_{\mathrm{I}}$ to $2 s$, $L_{\mathrm{II}, \mathrm{III}}$ to $2 p$, etc.

\begin{tabular}{|c|c|c|c|c|c|c|}
\hline & $\mathrm{ZnS}$ & $\mathrm{ZnSe}$ & $\mathrm{ZnTe}$ & $\mathrm{CdTe}$ & $\begin{array}{l}\text { Bearden } \\
\text { and } \\
\text { Burr }\end{array}$ & $\begin{array}{l}\text { Herman } \\
\text { and } \\
\text { Skillman }\end{array}$ \\
\hline $\operatorname{Zn}(3 p)$ & 90.9 & 90.6 & 89.4 & $\cdots$ & 86.6 & 91.6 \\
\hline $\mathrm{Cd}(4 p)$ & $\begin{array}{c}93.75 \\
\ldots .\end{array}$ & $\begin{array}{c}93.35 \\
\ldots\end{array}$ & $\begin{array}{c}93 \\
\ldots\end{array}$ & $\ddot{65}$ & $\cdots$ & $\begin{array}{l}94.8 \\
74.9\end{array}$ \\
\hline $\operatorname{Se}(3 d)$ & $\begin{array}{l}\ldots \\
\ldots \\
\ldots\end{array}$ & $\begin{array}{c}\ldots \\
59 \\
\ldots\end{array}$ & $\begin{array}{l}\ldots \\
\ldots \\
\ldots\end{array}$ & $\ldots$ & $\begin{array}{l}57 \\
\ldots\end{array}$ & $\begin{array}{l}80.4 \\
66 \\
67\end{array}$ \\
\hline
\end{tabular}

${ }^{22} \mathrm{~J}$. A. Bearden and A. F. Burr, Rev. Mod. Phys. 39, 125 (1967)

M. Cardona and G. Harbeke, Phys. Rev. 137, A1467 (1965). 


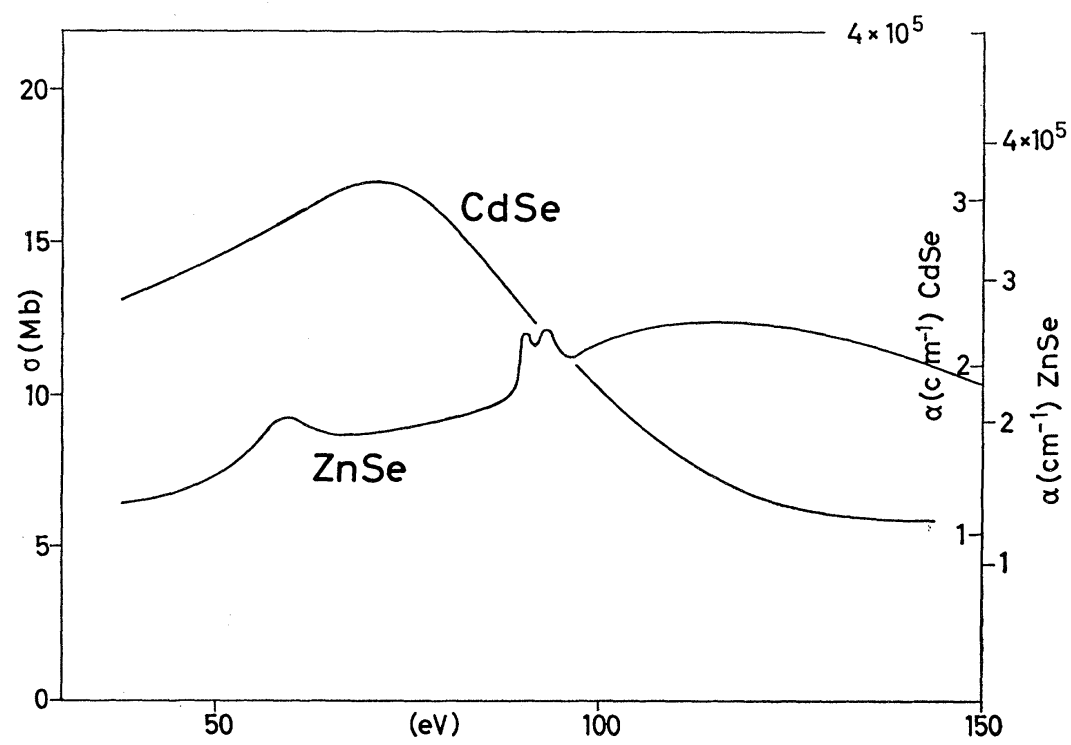

FIG. 2. Absorption spectrum of $\mathrm{ZnSe}$ and CdSe at room temperature in the soft-x-ray region.

the fundamental gap of $\mathrm{ZnS}$ is about $1.1 \mathrm{eV}$ higher than that of $\mathrm{ZnSe}$.

Figure 4 shows broad maxima with some superimposed structure for the absorption spectra of $\mathrm{ZnTe}$ and CdTe. The absorption coefficients at the maxima are considerably higher than those of Figs. 1 and 2. Some faint structure, corresponding roughly to the doublets

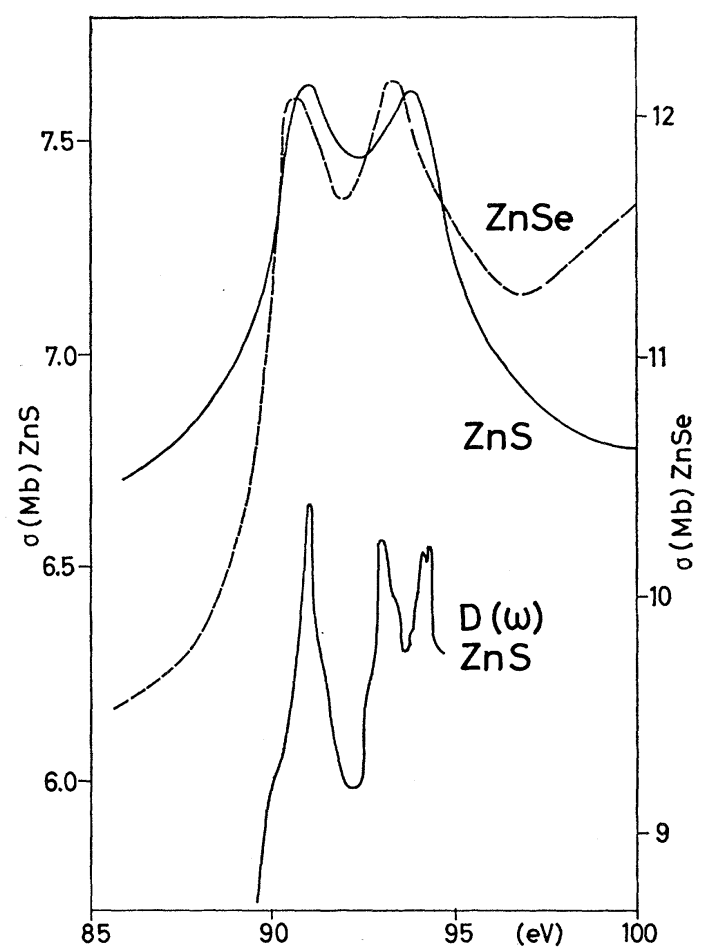

Fig. 3. Absorption doublet observed for $\mathrm{ZnS}$ and $\mathrm{ZnSe}$ in the $85-100-\mathrm{eV}$ region. This doublet is associated with the $M_{\mathrm{II}, \mathrm{III}}$ edge of $\mathrm{Zn}$. Also, density of conduction states (arbitrary scale and origin of energies) calculated by Herman et al. (Ref. 3) for $\mathrm{ZnS}$. of Fig. 3, can be seen near the maximum of the ZnSe spectrum (see Table I), but the assignment is somewhat arbitrary since more than a doublet is observed. Scattered light prevents, in view of the strong absorption background, the possibility of enhancing the observed fine structure by measuring thicker samples. The CdTe spectrum exhibits, beside some structure near the maximum, a hump at $65 \mathrm{eV}$ which, according to Table I, could be related to the $N_{\text {II,III }}$ edge of $\mathrm{Cd}$.

Figure 5 shows the absorption spectra of $\mathrm{PbS}$ and PbTe. No fine structure appears in these spectra; a change in slope in the $\mathrm{PbS}$ spectrum at $80 \mathrm{eV}$ could be related to the $\mathrm{O}_{\mathrm{II}, \mathrm{III}}$ threshold of $\mathrm{Pb}$ (see Table II). A compilation of the positions of the absolute maxima in the spectra of Figs. 1, 2, 4, and 5, together with the absorption cross sections at the maxima, is presented in Table III.

TABLE II. Energies (in eV) of (1) atomic core levels which may produce structure in the soft-x-ray spectra and (2) $x$-ray emission thresholds of the $\mathrm{Cd}, \mathrm{Zn}$, and $\mathrm{Pb}$ chalcogenides related to the spectral region of interest in this paper.

\begin{tabular}{ccccccc}
\hline \hline & \multicolumn{3}{c}{$d$ electrons } & \multicolumn{3}{c}{$p$ electrons } \\
& $(1)^{\mathrm{a}}$ & $(2)^{\mathrm{b}}$ & & $(1)^{\mathrm{a}}$ & $(2)^{\mathrm{b}}$ & \\
\hline $\mathrm{S}$ & $\cdots$ & $\cdots$ & $\cdots$ & 172 & 165 & $2 p$ \\
& $\cdots$ & $\cdots$ & & 173 & $\cdots$ & \\
$\mathrm{Se}$ & 66.1 & 57 & $3 d$ & 166 & 162 & $3 p$ \\
& 67.0 & 57 & & 172 & 168 & \\
$\mathrm{Te}$ & 54.5 & 40 & $4 d$ & 135 & 110 & $4 p$ \\
& 52.9 & 40 & & 126 & 110 & \\
$\mathrm{Zn}$ & 17.3 & 8.1 & $3 d$ & 91.6 & 87 & $3 p$ \\
& 17.7 & 8.1 & & 94.8 & 87 & \\
$\mathrm{Cd}$ & 18.8 & 9.3 & $4 d$ & 74.9 & 67 & $4 p$ \\
& 19.5 & 9.3 & & 80.4 & 67 & \\
$\mathrm{~Pb}$ & 32.9 & 19.2 & $5 d$ & 97.5 & 86 & $5 p$ \\
& 35.8 & 21.8 & & 115 & 86 & \\
\hline
\end{tabular}

a F. Herman and S. Skillman, Ref. 19. 
FIG. 4. Absorption spectra of $\mathrm{ZnTe}$ and $\mathrm{CdTe}$ at room temperature in the soft-x-ray region.

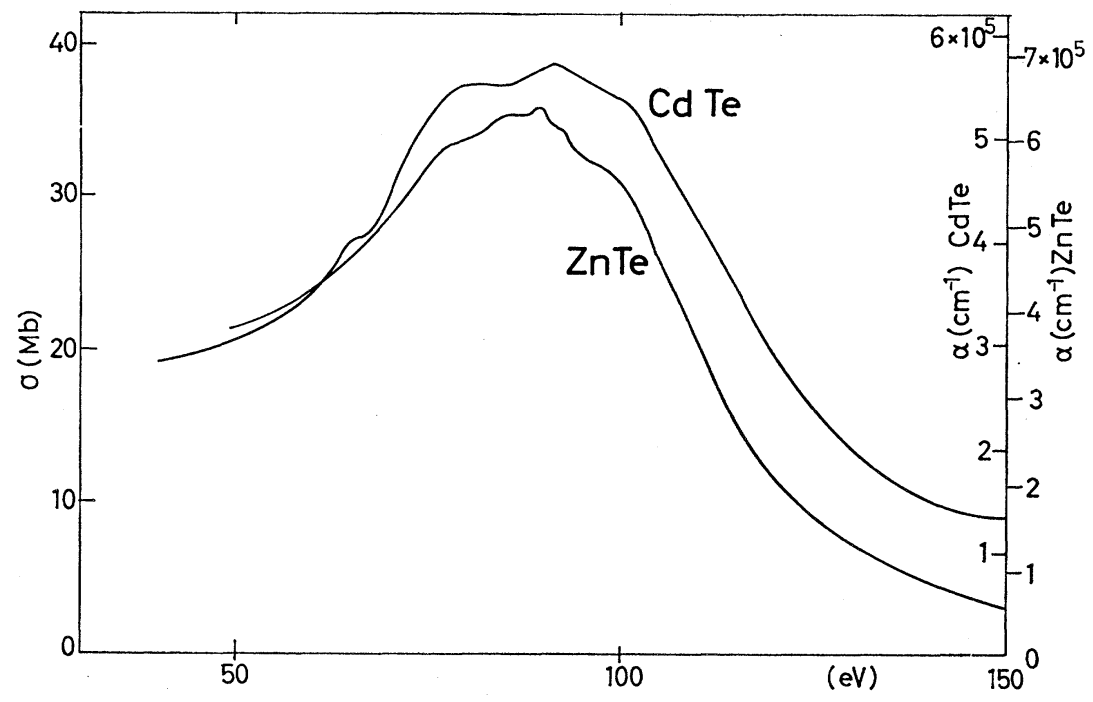

\section{DISCUSSION}

We show in Table II the x-ray emission thresholds and the energies of the atomic core levels which could produce optical structure in our photon energy region. As already mentioned, relatively narrow structure has been observed in the $\mathrm{Zn}$ compounds associated with the $M_{\text {II,III }}$ edge of $\mathrm{Zn}$, in CdTe associated with the $N_{\text {II,III }}$ edge of $\mathrm{Cd}$, and in $\mathrm{ZnSe}$ associated with the $M_{\mathrm{IV}, \mathrm{v}}$ edge of Se. The reason why such narrow structure does not appear for all materials measured can be associated with the existence of the broad maxima to be discussed later. The strength of the maxima (especially for the compounds of $\mathrm{Te}$ and $\mathrm{Pb}$ ), and possibly broadening due to interchannel interaction, ${ }^{16}$ prevent the appearance of such structure.
TABLE III. Effective number of electrons $N_{\text {eff }}$ whose oscillator strength is exhausted between 30 and $150 \mathrm{eV}$, energy $E_{\max }$ (in $\mathrm{eV}$ ), and strength $\left(\sigma_{\max }\right)$ of the absolute absorption maximum in the same region for several material considered here and for gaseous $\mathrm{Kr}$ and $\mathrm{Xe}$.

\begin{tabular}{lccc}
\hline \hline & $\begin{array}{c}N_{\text {eff }} \text { (electrons } \\
\text { per molecule) }\end{array}$ & $E_{\max }(\mathrm{eV})$ & $\sigma_{\max }(\mathrm{Mb})$ \\
\hline $\mathrm{ZnS}$ & 7.2 & 92 & 7.6 \\
$\mathrm{CdS}$ & 6.2 & 60 & 12.4 \\
$\mathrm{ZnSe}$ & 10.4 & 120 & 12.6 \\
$\mathrm{CdSe}$ & 11.5 & 70 & 16.9 \\
$\mathrm{ZnTe}$ & 18 & 89 & 35.5 \\
$\mathrm{CdTe}$ & 26.5 & 90 & 38.5 \\
$\mathrm{PbS}$ & 10.6 & 58 & 20 \\
$\mathrm{PbTe}$ & 22.5 & 82 & 34 \\
$\mathrm{Kr}$ & 2 & 200 & 3.5 \\
$\mathrm{Xe}^{\mathrm{a}}$ & 10 & 100 & 29 \\
\hline
\end{tabular}

a Obtained from Ref. 16.
FIG. 5. Absorption spectra of $\mathrm{PbTe}$ and $\mathrm{PbS}$ at room temperature in the soft-x-ray region.

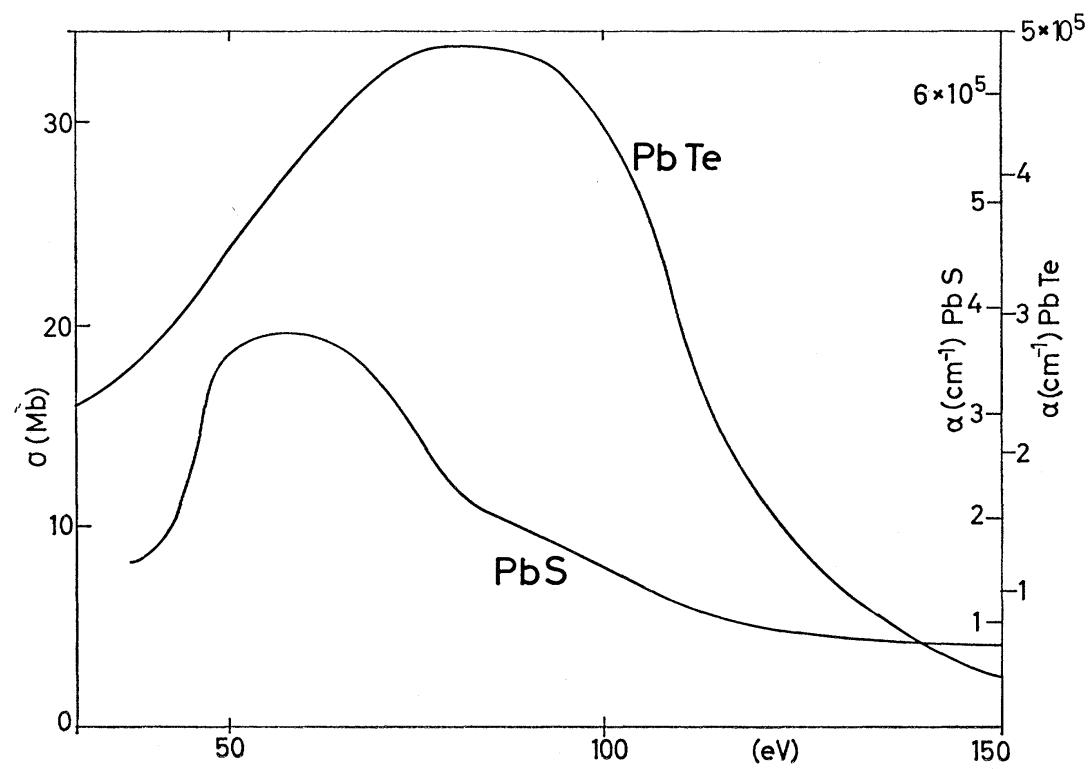




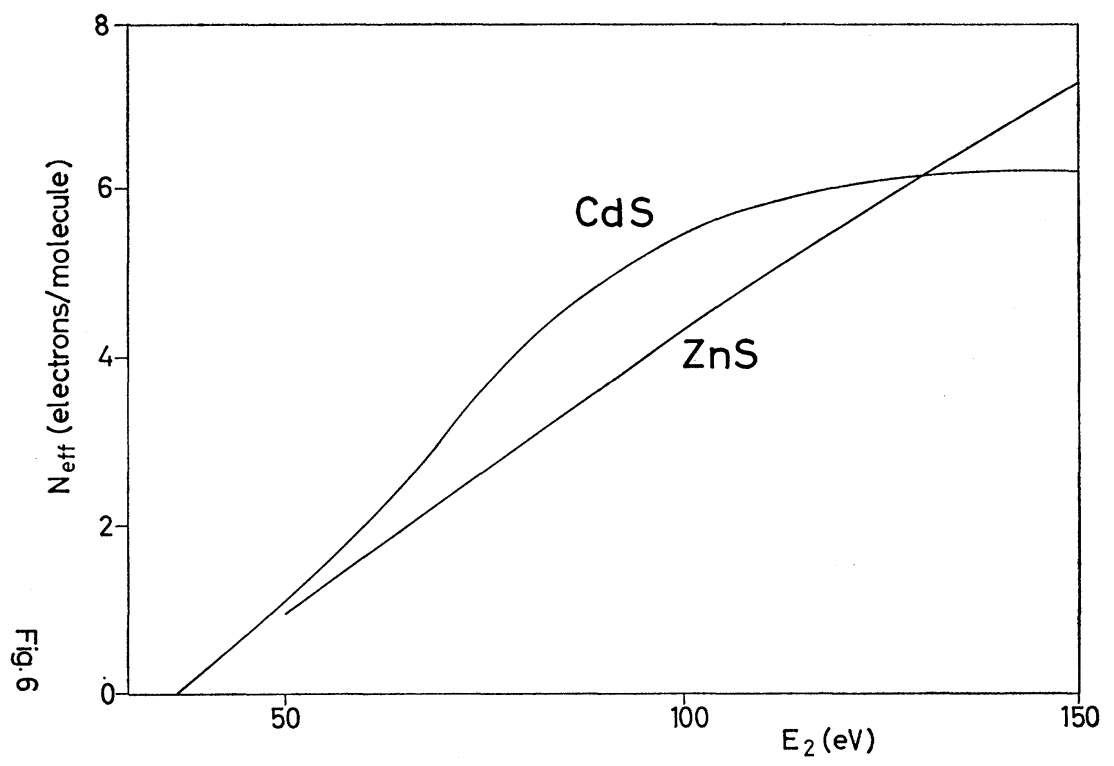

FIG. 6. Effective number of electrons whose oscillator strength is exhausted between $36 \mathrm{eV}$ and the energy $E_{2}$, for $\mathrm{CdS}$ and $\mathrm{ZnS}$.

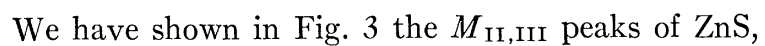
together with the density of conduction states calculated for this material. ${ }^{3}$ It is possible to speculate that the first peak (lower energy) of the experimental doublet is a reflection of the first peak in the density of states, while the observed higher-energy peak is the unresolved broadened version of the second and third peaks in the density of states. The spin-orbit splitting of the $d$ core levels, however, obliges us to expect the superposition of two similar density-of-states-type spectra separated by the spin-orbit energy $(\sim 3 \mathrm{eV})$. The low-energy component of this superposition should have a strength about $\frac{2}{3}$ of the high-energy component. Hence, a triplet, and not a doublet, would be expected from the density of states of Fig. 3 on the basis of the argument given above. One must, therefore, either conclude that one of the expected peaks is suppressed because of a small oscillator strength or that the observed spectra are atomiclike excitonic (Frenkel) excitations not bearing a direct relationship to the density of conduction states. We do not feel we can, at present, elucidate any further this question, which is of great interest in connection with the interpretation of fine structure also observed in this region for the alkali halides ${ }^{18}$ and the rare-gas solids. ${ }^{17}$

The broad, usually dominant, components of the observed spectra bear a strong similarity to the broad spectra observed for gaseous and solid $\mathrm{Kr}$ and $\mathrm{Xe}^{16,17}$ in the soft-x-ray region. Similar broad spectra have also been reported for the alkali iodides ${ }^{18}$ and for a number of heavy elements. ${ }^{24}$ For comparison, we have listed in Table III the energy of the broad maxima and its cross

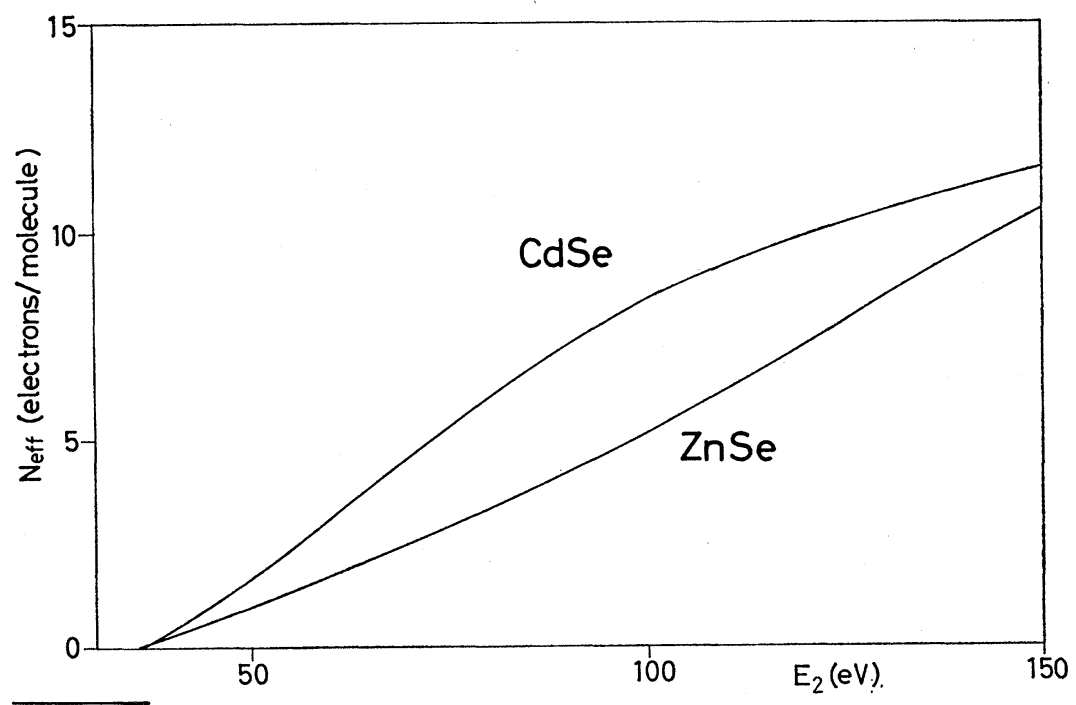

Fig. 7. Effective number of electrons whose oscillator strength is exhausted between $36 \mathrm{eV}$ and the energy $E_{2}$, for CdSe and $\mathrm{ZnSe}$.

${ }^{24}$ R. Haensel, C. Kunz, T. Sasaki, and B. Sonntag, Appl. Opt. 7, 301 (1968). 
FIG. 8. Effective number of electrons whose oscillator strength is exhausted between $36 \mathrm{eV}$ and the energy $E_{2}$, for CdTe and $\mathrm{ZnTe}$.

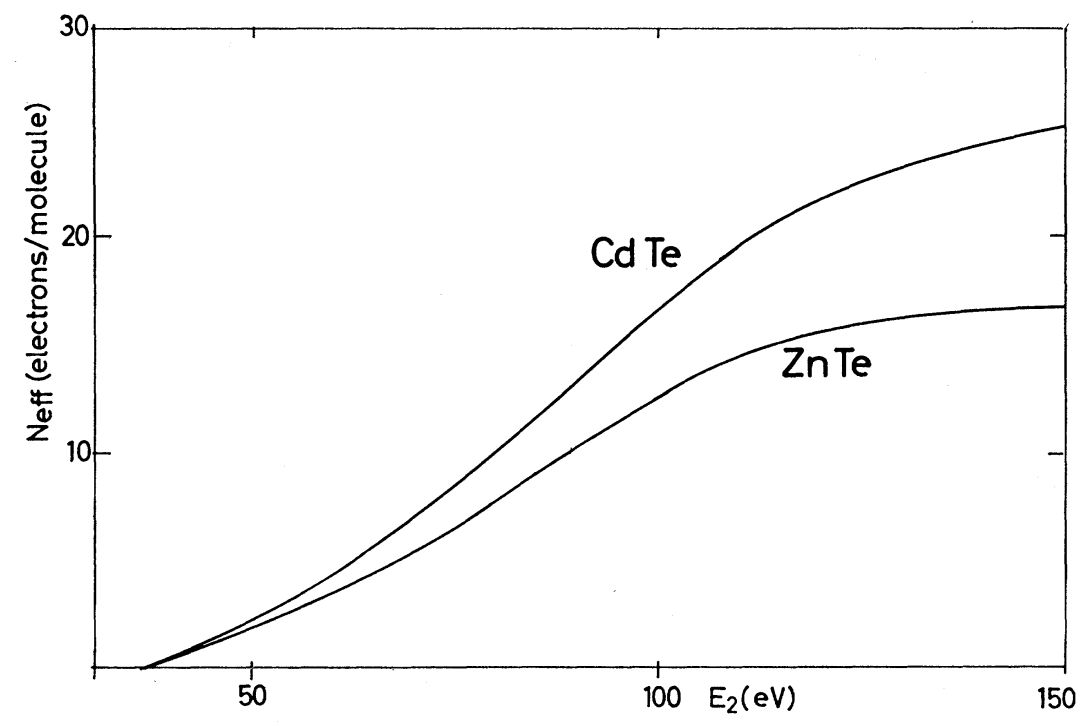

section for $\mathrm{Kr}$ and $\mathrm{Xe}$. These maxima in the atomic spectra have been attributed to $d \rightarrow f$ transitions, with the $f$ levels above the ionization threshold (sometimes referred to as delayed $d$ threshold). ${ }^{16}$ These transitions are particularly strong when there are unoccupied $f$ states belonging to the same shell as the initial filled $d$ levels (e.g., for the fifth and lower rows of the periodic table; $\mathrm{Cd}, \mathrm{Te}, \mathrm{Xe}, \mathrm{Pb})$. It is clearly apparent from Table III and our figures that the observed broad maxima are particularly strong for compounds with Te and $\mathrm{Pb}$, in agreement with the atomic argument given above. For reasons which we do not know, the corresponding maxima for the cadmium compounds are weaker. Recent work by Harrison et al. ${ }^{25}$ on the photoionization cross section of atomic Cd shows a broad maximum at an energy similar to that of CdS (Fig. 1) and with a comparable cross section $(\sim 15 \mathrm{Mb}$ at the maximum).

An examination of Tables II and III indicates that the broad maxima of all tellurides are mostly due to the $d \rightarrow f$ (same shell) transitions of Te. For PbTe, this maximum may be shifted to slightly lower energies because of the strong superposition of $d \rightarrow f$ transitions of $\mathrm{Pb}$ which have a maximum at $58 \mathrm{eV}$ (see $\mathrm{PbS}$ ). The thresholds of these $d$ transitions lie all at around $30 \mathrm{eV}$ and, hence, the delay of the $d \rightarrow f$ maximum is quite large. A detailed study of the threshold region (below $36 \mathrm{eV})$, to be performed at a later date, is desirable for the sake of completeness. The weaker broad spectrum of CdSe seems dominated by the $d \rightarrow f$ transitions (same shell also) of $\mathrm{Cd}$ (see also $\mathrm{CdS}$ ). That of ZnSe, with a maximum at $120 \mathrm{eV}$, should be a superposition
FIG. 9. Effective number of electrons whose oscillator strength is exhausted between $36 \mathrm{eV}$ and the energy $E_{2}$, for $\mathrm{PbS}$ and $\mathrm{PbTe}$.

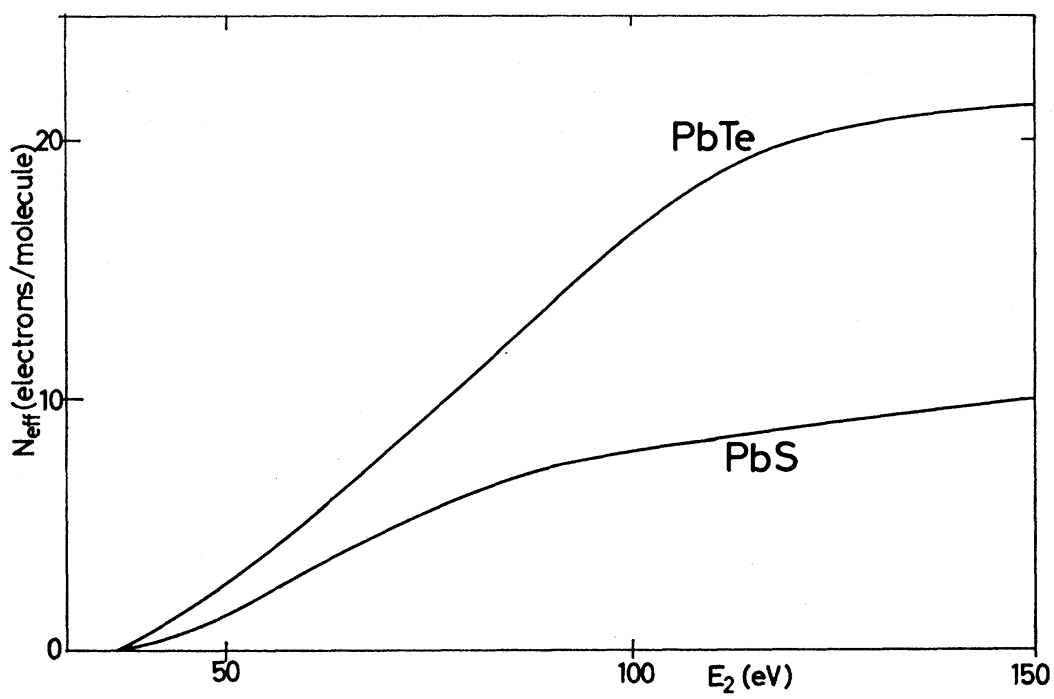

${ }^{25}$ H. Harrison, R. I. Schoen, K. E. Schubert, and R. B. Cairns, J. Chem. Phys. (to be published). 
of $d \rightarrow f$ transitions of $\mathrm{Zn}$ and Se plus $p \rightarrow d$ transitions of $\mathrm{Zn}$ : It is weaker than for the tellurides and for $\mathrm{CdSe}$ since all the transitions mentioned have initial and final states in different atomic shells.

For a more quantitative treatment of the broad parts of the spectra, it is convenient to calculate the effective density of states whose oscillator strength has been exhausted between energies $E_{1}$ and $E_{2}{ }^{24,26}$ :

$N_{\text {eff }}($ electrons/molecule $)$

$$
\begin{aligned}
& =2.3 \times 10^{15} a^{3} \int_{E_{1}}^{E_{2}} \alpha(E) n(E) d E \\
& =9.1 \times 10^{15} \int_{E_{1}}^{E_{2}} n(E) \sigma(E) d E
\end{aligned}
$$

In Eq. (1), $a$ is the lattice constant and $n(E)$ is the real part of the refractive index. A calculation of the imaginary part of the refractive index $k(E)$ from the observed absorption coefficients indicates that $n(E)$ differs from 1 by no more than $10 \%$ (the maximum values of $n$ should be similar to those of $k$ for the observed line widths). In view of this and of the qualitative meaning of $N_{\text {eff }}$, we set $n(E)=1$ for the evaluation of Eq. (1) from our experimental values of $\alpha(E)$. The values of $N_{\text {eff }}$ calculated with $E_{1}=37 \mathrm{eV}$ are shown in

${ }^{26}$ H. R. Philipp and H. Ehrenreich, Phys. Rev. 129, 1550 (1963).
Figs. 6-9 as a function of $E_{2}$. The effective number of electrons per molecule whose oscillator strength is exhausted in our experimental region, i.e., the ordinates of Fig. 6-9 for $E=150 \mathrm{eV}$, are tabulated under $N_{\text {eff }}$ in Table III. It is clear from this table that $N_{\text {eff }}$ amounts to all of the $5 d$ electrons of $\mathrm{Pb}$ for $\mathrm{PbS}$ and these electrons plus the $4 d$ electrons of Te for $\mathrm{PbTe}$. For $\mathrm{CdTe}$ and $\mathrm{ZnTe}$ also, practically all of the outermost core $d$ electrons are exhausted in our experimental region (slightly less for $\mathrm{ZnTe}$ ). This number becomes smaller for the selenides, undoubtedly related to the fact that the $d \rightarrow f$ transitions of Se do not take place within the same shell. About 6 electrons/atom are exhausted for CdS in constrast to 7 electrons/atoms for $\mathrm{ZnS}$. We must point out, however, that for these two materials the error in the determination of the film thickness may be as high as $30 \%$ (see Sec. II). Thus, an error of $30 \%$ in $N_{\text {eff }}$ is possible for both $\mathrm{ZnS}$ and CdS.

\section{ACKNOWLEDGMENTS}

We would like to thank Peter Rabe for the construction of the equipment for in situ evaporation, Günter Singmann for help in the preparation of the samples and substrates, and Dr. Bernd Sonntag for a number of valuable discussions. Thanks are also due Dr. R. I. Schoen for sending us a preprint of his paper (Ref. 25) prior to publication and to the Deutsche Forschungsgemeinschaft for continuing support of this paper. 